\title{
Performance Status, Prognostic Scoring and Parenteral Nutrition Requirements Predict Survival in Patients with Advanced Cancer Receiving Home Parenteral Nutrition
}

\author{
Niamh Keane; ${ }^{* 1}$ Konstantinos C. Fragkos; ${ }^{* 1}$ Pinal S. Patel; ${ }^{1}$ Friderike Bertsch; ${ }^{1}$ Shameer J. Mehta; ${ }^{1}$ \\ Simona Di Caro; ${ }^{1}$ Farooq Rahman ${ }^{1}$ \\ ${ }^{1}$ Intestinal Failure Service, Gastrointestinal (GI) Services, University College London Hospitals NHS \\ Foundation Trust, London UK \\ ${ }^{*}$ These authors contributed equally to this manuscript
}

\begin{abstract}
We describe a cohort of Home Parenteral Nutrition (HPN) patients with advanced cancer in order to identify factors affecting prognosis. Demographic, anthropometric, biochemical and medical factors, Karnofsky Performance Status (KPS), Glasgow Prognostic Score (GPS), and PN requirements were recorded. Univariate and multivariate analyses were performed including Kaplan-Meier curves, Cox Regression and correlation analyses. In total, 107 HPN patients (68 women, 39 men, mean age 57 years) with advanced cancer were identified. The main indications for HPN were bowel obstruction (74.3\%) and high output ostomies (14.3\%). Cancer cachexia was present in $87.1 \%$ of patients. The hazard ratio (HR) for upper gastrointestinal and 'other' cancers vs gynaecological malignancy was $1.75(\mathrm{p}=0.077)$ and $2.11(\mathrm{p}=0.05)$, respectively. KPS score, GPS,

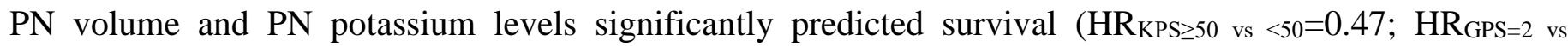
GPS $=0=3.19$ ). In multivariate analysis, KPS and GPS remained significant predictors $(\mathrm{p}<0.05)$, whilst PN volume reached borderline significance $(\mathrm{p}=0.094)$. Survival was not significantly affected by the presence of metastatic disease, previous or concurrent surgery, chemo-radiotherapy, or indication for HPN ( $>>0.05)$. Most patients passed away in their homes or hospice (77.9\%). Performance status, prognostic scoring and PN requirements may predict survival in patients with advanced cancer receiving HPN.
\end{abstract}

Keywords: parenteral nutrition, advanced cancer, bowel obstruction, performance status, survival, clinical

\author{
$\underline{\text { Abbreviations }}$ \\ CI: confidence Interval \\ CRP: C-Reactive Protein \\ ESPEN: European Society for Clinical Nutrition and Metabolism \\ GPS: Glasgow Prognostic Score \\ HPN: home parenteral nutrition \\ HR: hazard ratio \\ IQR: Interquartile range \\ KPS: Karnofsky Performance Status \\ PN: parenteral nutrition \\ SD: standard deviation \\ UCLH: University College London Hospitals \\ WCC: White cell count
}




\section{Introduction}

Artificial nutrition may be indicated in malnourished oncology patients who cannot autonomously meet their nutritional requirements (1). In the last decade there has been an increased trend in referrals and this cohort currently represent the commonest indication for home parenteral nutrition (HPN) worldwide. Nevertheless, the use of long-term HPN in advanced/incurable (previously referred to as palliative) cancer patients remains controversial in the UK (1-6). Timely commencement of HPN is considered in advanced cancer patients with an acceptable quality of life who would die from malnutrition-related complications (and in some cases starvation) before tumour progression $(3,5,7)$. Cachexia is prevalent in more than $50 \%$ of patients with advanced cancer and can lead to death in more than $10 \%$ of subjects (8-10).

Discrepancies in worldwide clinical practice relate to financial and ethical issues, differing clinician views, socio-cultural attitudes and religious beliefs. Appropriate and careful selection of patients to offer HPN is a crucial point for clinicians. It is generally accepted that HPN should not be considered in imminently dying patients. However, other cases are less clear and robust criteria and guidelines are lacking. Financially, it is difficult to estimate direct and indirect costs of HPN services considering the variation between countries. Nevertheless, many advocate that its use in this patients' cohort constitutes an unacceptable additional burden to already overstretched health economies $(2,4-6,11)$. Finally, there is a paucity of high quality evidence about the true impact of HPN on survival, tumour progression, performance status and quality of life to guide clinical practice in advanced cancer, as randomised controlled trials would be unethical (2, 4-6, 11, 12).

Prevalence of HPN in patients with advanced cancers throughout the world reflects differing practices $(2,6$, 13-15). For example, in Denmark and the UK the prevalence is less than $10 \%$ while Netherlands, Italy and the USA are greater than $50 \%(2,5-7,11,16,17)$. The European Society for Clinical Nutrition and Metabolism (ESPEN) guidelines (Grade C) suggest considering HPN in advanced cancer patients with intestinal failure if enteral nutrition is insufficient to meet nutritional requirements; expected survival due to tumour progression is over two to three months; it is expected that PN will stabilise or improve performance status and/or quality of life; and the patient desires PN $(1,7)$. The commonest indication for HPN in patients with advanced cancer is bowel obstruction, caused by intra- or extra-abdominal malignancy with intraperitoneal spread, which is usually subacute $(1,6)$. Although contentious, HPN may facilitate survival and augment the effectiveness of palliative chemo-radiotherapy. However, some authors contend that HPN is a burden to patients and possibly feeds the tumour $(2,6,18)$.

Robust practical guidance is lacking in advanced cancer patients on HPN despite previous studies investigating prognostic factors $(2,3,6,13,19-28)$. In the present study, we aim to examine the prognostic significance of performance status, type and site of tumour, previous or concurrent chemo-radiotherapy, anthropometric characteristics, nutritional and inflammatory status, demographic characteristics, serum biochemistry and prognostic indices based on a large cohort of patients with advanced cancer receiving HPN at University College London Hospitals (UCLH), UK.

\section{Methods}

\section{Settings}

UCLH is a 665-bed tertiary hospital in central London, UK, and a national referral centre for many types of cancer. HPN candidates were initially identified by primary teams (oncology, gynaecology, urology, gastroenterology, surgery) and referred to the multi-disciplinary Nutrition Support Team. In each case, the options and prognosis were carefully reviewed and discussed with the primary team and patient before initiating HPN. Follow-up was performed regularly (every 4-6 weeks) and as clinically relevant. 


\section{Population}

All adult patients, 18 years or older, with advanced cancer discharged on HPN from UCLH from $1^{\text {st }}$ of January 2006 to $15^{\text {th }}$ of October 2016 were included in the analysis. Loss to follow-up was the single exclusion criterion. Data was collected retrospectively through UCLH electronic patients' records and the HPN databases held by the Nutrition Support team.

Data Collection

The following data were collected:

Demographics and Medical Factors: primary malignancy, presence of metastases (defined as disease at nonadjacent organs), surgery for malignancy, chemotherapy and/or radiotherapy before or during PN, main indication for HPN and the place of death.

HPN Requirements: duration of HPN, volume, nitrogen, glucose, lipid and lipid formulation, electrolytes and days per week of HPN. Patients received individualised formulae containing fluids, amino acids, glucose, lipids, minerals and trace elements.

Serum Biochemistry: C-Reactive Protein (CRP), white cell count (WCC), albumin, haemoglobin, sodium, potassium, magnesium, phosphate and adjusted calcium.

Performance Status: Karnofsky Performance Status (KPS) was assessed by the attending clinician and a score of 50 was used as cut-off for analyses, with over 50 indicating that the patient is unable to work but able to live at home and care for most personal needs with varying amount of assistance needed $(29,30)$.

Prognostic Scoring: Glasgow Prognostic Score (GPS) was calculated and is based on the serum levels of albumin and CRP. A score of 2 is allocated when both an elevated CRP level (>10 mg/L) and hypoalbuminemia $(<35 \mathrm{~g} / \mathrm{L})$ are present $(31)$.

Anthropometric Characteristics: Habitual body weight, height, habitual BMI, weight upon starting PN, percentage of weight loss when PN commenced (from usual weight), BMI when PN commenced, weight at follow-up outpatient appointments at 3 and 6 months after discharge. Patients were defined as having cancer cachexia if weight loss $>5 \%$ (in absence of simple starvation) was reported or BMI $<20 \mathrm{~kg} / \mathrm{m}^{2}$ and any degree of weight loss $>2 \%$ (32).

Timeline: The day that HPN started (i.e. discharge date or baseline date) was considered as the starting time point for all measurements including anthropometrics, blood tests, and HPN requirements. End time point was date of death or censoring date (15 October 2016). Survival was reported in weeks.

\section{Ethical Considerations}

Individual patient data were anonymized and regulatory approval was granted by the site institutional review board. This study involved the collection of existing data and records. Informed consent was exempted according to the decision of institutional ethics board committees. This is a retrospective study and adherence to the principles of the Declaration of Helsinki was followed during design and analysis.

\section{Statistical Analysis}

Data are presented as mean and standard deviation (SD) or median and interquartile range (IQR) for continuous data and absolute and relative frequency for categorical data, respectively. Overall survival was calculated from the onset of HPN until death irrespective of cause or censoring date on 15 October 2016. Univariate analyses were conducted with chi-square test, Spearman's rho for correlations, t-tests and ANOVA. After Kaplan-Meier analysis on the impact of the variables on survival, the groups were compared with the log-rank test. Variables that showed a $p$-value of $\leq 0.05$ in the univariate analysis were included into Cox regression analyses. A $p$-value $\leq 0.05$ was considered significant. For data analysis, IBM SPSS Statistics (Release 22.0.0. 2010, Chicago (IL), USA: SPSS, Inc., an IBM Company) was used. 


\section{Results}

\section{Patient Characteristics}

Demographics and Medical Factors. Our sample included 107 patients (68 females, 39 males) with mean age $57 \pm 12$ years. Malignancy types and frequencies are shown in Table 2. Most patients had metastatic disease (81.3\%) and had undergone surgery for their malignancy (79\%), or chemotherapy before and/or during PN administration $(90.4 \%)$. The majority of patients were radiotherapy naive $(71.2 \%)$. Most patients passed away in their homes or hospice $(77.9 \%$ ) (Table 1$)$.

HPN characteristics. The majority of patients had solely HPN $(97.1 \%)$, while $3 \%$ required additional intravenous fluids. Median duration of HPN was 12.9 weeks (5.6-29.0). The main indications for HPN were bowel obstruction (74.3\%) and high output ostomies (14.3\%). The majority of patients were PN-dependent until death (Table 1). Average HPN requirements are shown in Table 3.

Anthropometrics. Mean BMI was $19.9 \pm 4.1 \mathrm{~kg} / \mathrm{m}^{2}$ with $41.8 \%$ of patients being underweight $\mathrm{BMI} \leq 18.5$ $\mathrm{kg} / \mathrm{m}^{2}$ ). Documented weight loss was greater than $10 \%$ and $30 \%$ in $58.1 \%$ and $11.3 \%$ of patients, respectively. Cancer cachexia was present in $87.1 \%$. Mean weight on discharge was $55.3 \pm 12.3 \mathrm{~kg}$ with a relative increase up to $58.6 \pm 11.3 \mathrm{~kg}$ at 6 months in the outpatient setting (Table 1).

Performance status and prognostic score. Mean KPS score was $50 \pm 16 ; 66 \%$ of patients scored KPS 50 to 80. GPS was 2 for most patients (34.6\%) (Table 1$)$.

Serum Biochemistry. On discharge, mean CRP was high at $46 \pm 48.3 \mathrm{mg} / \mathrm{L}$ (normal range 0-5.0) with normal WCC $\left(9.6 \pm 6.8 \times 10^{9} / \mathrm{L}\right.$, normal range 3.0-10.0) and albumin borderline low at $32.2 \pm 6 \mathrm{~g} / \mathrm{L}$ (normal range 34-50). Mean haemoglobin was slightly below normal (101.6 $\pm 15 \mathrm{~g} / \mathrm{L}$, normal range 115-155) and mean values of electrolytes were within normal values (Table 1).

Table 1. Patient Characteristics.

\begin{tabular}{lc}
\hline Variables & Results \\
\hline General Characteristics & \\
\hline Age $(\text { years })^{*}$ & $3(2.8)$ \\
$\leq 35$ & $24(22.4)$ \\
$36-49$ & $48(44.9)$ \\
$50-64$ & $30(28)$ \\
$65-79$ & $2(1.9)$ \\
$\geq 80$ & \\
Gender & $68(63.6)$ \\
Female & $39(36.4)$ \\
Male & \\
Type of malignancy ${ }^{*} \dagger$ & $37(34.6)$ \\
Gynaecological & $21(19.6)$ \\
Upper Gastrointestinal & $24(22.4)$ \\
Lower Gastrointestinal & $10(9.3)$ \\
Hepato-pancreatobiliary & $5(4.7)$ \\
Haematological & $10(9.3)$ \\
Other & \\
GPS & $10(9.3)$ \\
0 & $19(17.8)$ \\
1 & \\
\hline
\end{tabular}




\begin{tabular}{|c|c|}
\hline Variables & Results \\
\hline 2 & $37(34.6)$ \\
\hline Not available (n/a) & $41(38.3)$ \\
\hline \multicolumn{2}{|l|}{ HPN Characteristics } \\
\hline \multicolumn{2}{|l|}{ HPN Indication $^{*}$} \\
\hline Bowel Obstruction & $78(74.3)$ \\
\hline High Output Ostomy & $15(14.3)$ \\
\hline Other ${ }^{*}$ & $12(11.4)$ \\
\hline \multicolumn{2}{|l|}{ HPN days per week ${ }^{*}$} \\
\hline 4 & $5(4.7)$ \\
\hline 5 & $2(1.9)$ \\
\hline 7 & $100(93.4)$ \\
\hline \multicolumn{2}{|l|}{ PN lipid type } \\
\hline SMOF & $34(38.2)$ \\
\hline Other ${ }^{\S}$ & $55(61.8)$ \\
\hline \multicolumn{2}{|l|}{ HPN termination reason ${ }^{*}$} \\
\hline Death & $93(94.9)$ \\
\hline Established on oral / enteral feeding & $4(4.1)$ \\
\hline Patient declined & $1(1)$ \\
\hline
\end{tabular}

\begin{tabular}{|c|c|}
\hline Anthropometrics & \\
\hline Usual weight $(\mathrm{kg})^{\mathbb{T I}}$ & $65.3 \pm 14.6(n=65)$ \\
\hline Height $(m)^{\mathbb{I}}$ & $1.7 \pm 0.1(n=71)$ \\
\hline Usual BMI $\left(\mathrm{kg} / \mathrm{m}^{2}\right)^{\mathrm{II}}$ & $23.5 \pm 4.8(n=63)$ \\
\hline Weight upon starting $P N(\mathrm{~kg})^{\mathrm{II}}$ & $55.3 \pm 12.3(\mathrm{n}=71)$ \\
\hline$\%$ weight loss upon starting $P N^{\mathrm{II}}$ & $15.2 \pm 11.5(n=62)$ \\
\hline \multicolumn{2}{|l|}{$\%$ weight loss upon starting $H P N^{*}$} \\
\hline$<20 \%$ & $42(67.7)$ \\
\hline $20 \%-30 \%$ & $13(21)$ \\
\hline$>30 \%$ & $7(11.3)$ \\
\hline BMI upon starting $P N\left(\mathrm{~kg} / \mathrm{m}^{2}\right)^{\text {II }}$ & $19.9 \pm 4.1(n=67)$ \\
\hline Weight in outpatient clinic, 0-3 months $\left.(\mathrm{kg})^{\mathrm{I}}\right)$ & $54.5 \pm 9(n=37$ \\
\hline Weight in outpatient clinic, 3-6 months $(\mathrm{kg})^{4 l}$ & $58.6 \pm 11.3(\mathrm{n}=19)$ \\
\hline \multicolumn{2}{|l|}{ Serum Biochemistry } \\
\hline Sodium $(\mathrm{mmol} / \mathrm{L}, 135-145)^{\mathbb{T l}}$ & $136 \pm 4(n=66)$ \\
\hline Potassium (mmol/L, 3.5-5.1) $)^{\mathrm{I}}$ & $4.4 \pm 0.5(n=66)$ \\
\hline Magnesium $(\mathrm{mmol} / \mathrm{L}, 0.60-1.00)^{\mathrm{TI}}$ & $0.80 \pm 0.10(n=63)$ \\
\hline Phosphate (mmol/L, 0.87-1.45) $)^{\mathrm{II}}$ & $1.20 \pm 0.20(n=65)$ \\
\hline Adjusted calcium (mmol/L, 2.20-2.60) $)^{\mathrm{II}}$ & $2.40 \pm 0.10(n=66)$ \\
\hline
\end{tabular}

${ }^{*} \mathrm{~N}(\%)$

† Gynaecological: breast, ovarian, endometrial, cervical; Lower gastrointestinal: small bowel, appendix, colon, sigmoid, rectal, anal; upper gastrointestinal: oesophageal, gastric, duodenal; hepato-pancreatobiliary: liver, pancreas, bile ducts; haematological: leukaemia, lymphoma; others: peritoneal, bladder, sarcoma, thyroid.

* This included mucositis, graft-versus-host disease, radiation-related bowel disease, intra-abdominal collection, enterocutaneous fistula, malabsorption, intolerance or inability of enteral feeding.

$\S$ This included Clinoleic, Intralipid, Lipofundin and Triomel.

$\pi$ Mean \pm SD 
Survival: Univariate Analyses and Cox Regression

Overall mean survival was 30.8 weeks (95\% CI 21.4-39.6) and median survival was 14 weeks (IQR 5-34). At the time of analysis, $88.8 \%$ had passed away, 3-month survival was $49.5 \%$ and 6-month survival was $30.8 \%$. Univariate analyses are shown in Table 4. Kaplan-Meier curves for type of malignancy, HPN indication, KPS score, GPS, PN volume, and PN potassium content are shown in Figure 1 and for the rest of the variables in the Supplementary Materials (Supplementary Figures 1 and 2). Post-hoc power analyses were performed for the current sample size and significance level 0.05 , revealing power over $80 \%$ for most predictors in the present sample.

Demographics: Age and gender did not significantly predict survival $(p>0.05)$ (Table 2).

Medical factors: The incidence of death in patients with upper gastrointestinal $(\mathrm{HR}=1.75, p=0.077)$ and 'other' malignancies ( $\mathrm{HR}=2.11, p=0.05$ ) was significantly higher compared to patients with gynaecological malignancy. Metastatic disease, surgery, chemo-radiotherapy, or indication for HPN did not significantly affect survival $(p>0.05)$.

Performance status and prognostic scoring: KPS score and GPS significantly predicted survival. A higher KPS score was associated with longer survival (median survival was 20 weeks $v s 5$ weeks, for KPS $\geq 50$ vs KPS $<50$ respectively; HR $=0.47, p=0.001]$. A GPS of 0 was associated with longer survival than a GPS of 2 (median survival: 55 weeks vs 10 weeks, respectively; HR $=3.19, p=0.006$ ).

Anthropometrics: Weight, height, and BMI did not significantly correlate with survival.

Serum Biochemistry: The only significant predictors of survival were CRP (HR $=1.01, p=0.001)$ and WCC $(\mathrm{HR}=1.06, p=0.011)$. There was a trend suggesting improved survival in patients with a serum sodium above $135 \mathrm{mmol} / \mathrm{L}(\mathrm{HR}=0.62, p=0.097)$ and higher albumin levels $(\mathrm{HR}=0.96, p=0.052)$.

HPN requirements. Daily PN volume and potassium content predicted overall survival. An HPN volume of 2 L/day or more was associated with a significantly increased incidence of death (86\%) compared to less than 2 L/day (median survival: 12 vs 34 weeks, respectively). An HPN potassium of $60 \mathrm{mmol} / \mathrm{day}$ or more was associated with a significantly increased incidence of death (81\%) compared to a potassium content less than $60 \mathrm{mmol} / \mathrm{day}$ (median survival 10 vs 24 weeks, respectively). The type of HPN lipid (SMOFLipid (33) vs other) did not predict survival.

All statistically significant predictors associated with survival at univariate analysis were entered into a multivariate Cox regression model (Table 2). After adjusting for confounders (PN volume and PN potassium content), only KPS and GPS remained significant predictors of survival, whilst HPN volume reached borderline significance $(p=0.087)$. Higher scores of KPS and lower GPS were both associated with better survivals.

Spearman's rho correlations of survival with CRP $($ rho $=-0.484)$ and GPS (rho $=-0.444)$ indicated a moderate association, while WCC (rho $=-0.300)$, albumin (rho $=0.338)$, KPS (rho $=0.374)$, and PN potassium content (rho $=-0.247)$ were weakly correlated with survival. All correlations were significant $(p<0.05)$ (Supplementary Table 1).

Table 2. Survival times and Cox regressions.

\begin{tabular}{ll|lr|lc}
\hline Variables & Survival (weeks) & \multicolumn{2}{|l|}{ Univariate Cox regression } & \multicolumn{2}{|l}{ Multivariate Cox regression } \\
\hline & Median (IQR) & HR (95\% CI) & $\boldsymbol{p}$ & HR (95\% CI) & $\boldsymbol{p}$ \\
\hline Demographic characteristics & & & & \\
\hline Age (years) & & & & -- \\
$\quad<50$ & $16(9-36)$ & Ref. & & - \\
$50-64$ & $10(5-29)$ & $1.08(0.66-1.77)$ & 0.755 & -- & - \\
$\geq 65$ & $14(5-29)$ & $1.02(0.59-1.77)$ & 0.938 & -- & \\
Gender & & & & & \\
\hline
\end{tabular}


Keane et al. 7

\begin{tabular}{|c|c|c|c|c|c|}
\hline \multirow[t]{2}{*}{ Variables } & \multirow{2}{*}{$\begin{array}{l}\text { Survival (weeks) } \\
\text { Median (IQR) }\end{array}$} & \multicolumn{2}{|c|}{ Univariate Cox regression } & \multicolumn{2}{|c|}{ Multivariate Cox regression } \\
\hline & & HR $(95 \%$ CI $)$ & $p$ & HR $(95 \%$ CI $)$ & $p$ \\
\hline Female & $16(5-34)$ & Ref. & & -- & -- \\
\hline Male & $11(5-32)$ & $1.22(0.80-1.86)$ & 0.358 & -- & -- \\
\hline \multicolumn{6}{|l|}{ Medical Factors } \\
\hline \multicolumn{6}{|l|}{ Type of malignancy } \\
\hline Gynaecological & $14(5-55)$ & Ref. & & -- & -- \\
\hline Upper Gastrointestinal & $10(4-34)$ & $1.75(0.94-3.26)$ & 0.077 & -- & -- \\
\hline Lower Gastrointestinal & $12(9-29)$ & $1.38(0.80-2.40)$ & 0.251 & -- & -- \\
\hline Hepatobiliary & $18(10-43)$ & $1.21(0.59-2.52)$ & 0.603 & -- & -- \\
\hline Haematological & $17(9-48)$ & $0.95(0.33-2.71)$ & 0.916 & -- & -- \\
\hline Other & $10(3-23)$ & $2.11(1.00-4.45)$ & 0.050 & -- & -- \\
\hline \multicolumn{6}{|l|}{ Metastatic disease } \\
\hline No & $18(9-48)$ & Ref. & & -- & -- \\
\hline Yes & $12(5-32)$ & $1.38(0.79-2.39)$ & 0.259 & -- & -- \\
\hline \multicolumn{6}{|l|}{ Surgery } \\
\hline No & $16(7-23)$ & Ref. & & -- & -- \\
\hline Yes & $14(5-36)$ & $0.84(0.50-1.41)$ & 0.500 & -- & -- \\
\hline \multicolumn{6}{|l|}{ Chemotherapy } \\
\hline No & $23(16-29)$ & Ref. & & -- & -- \\
\hline Yes & $12(6-35)$ & $0.92(0.47-1.79)$ & 0.797 & -- & -- \\
\hline \multicolumn{6}{|l|}{ Radiotherapy } \\
\hline No & $14(6-29)$ & Ref. & & -- & -- \\
\hline Yes & $15(5-69)$ & $0.65(0.40-1.060$ & 0.086 & -- & -- \\
\hline \multicolumn{6}{|l|}{ HPN indication } \\
\hline Bowel obstruction & $12(6-29)$ & Ref. & & -- & -- \\
\hline High output ostomy & $23(5-55)$ & $0.88(0.49-1.56)$ & 0.659 & -- & -- \\
\hline Other & $30(9-54)$ & $0.61(0.31-1.230$ & 0.170 & -- & -- \\
\hline \multicolumn{6}{|l|}{ Anthropometrics } \\
\hline Usual weight (kg) & & $1.002(0.98-1.02)$ & 0.815 & -- & -- \\
\hline Height (m) & & $12.6(0.53-297.49)$ & 0.117 & -- & -- \\
\hline Usual BMI $\left(\mathrm{kg} / \mathrm{m}^{2}\right)$ & & $0.97(0.92-1.04)$ & 0.420 & -- & -- \\
\hline Weight upon starting $P N(\mathrm{~kg})$ & & $1.01(0.99-1.04)$ & 0.192 & -- & -- \\
\hline \multicolumn{6}{|l|}{$\%$ weight loss upon starting $H P N$} \\
\hline$<20 \%$ & $14(4-35)$ & Ref. & & -- & -- \\
\hline between $20 \%$ and $30 \%$ & $9(7-25)$ & $1.02(0.52-1.99)$ & 0.956 & & -- \\
\hline$\geq 30 \%$ & $30(9-103)$ & $0.64(0.26-1.55)$ & 0.325 & & -- \\
\hline \multicolumn{6}{|l|}{$B M I\left(\mathrm{~kg} / \mathrm{m}^{2}\right)$ upon starting $H P N$} \\
\hline $\mathrm{BMI} \leq 18.5$ & $15(7-32)$ & Ref. & & -- & -- \\
\hline $\mathrm{BMI}>18.5$ & $16(4-35)$ & $1.03(0.61-1.74)$ & 0.921 & -- & -- \\
\hline \multicolumn{6}{|l|}{ Cancer cachexia } \\
\hline No & $14(8-29)$ & Ref. & & -- & -- \\
\hline Yes & $14(6-35)$ & $1.09(0.49-2.43)$ & 0.827 & -- & -- \\
\hline $\begin{array}{l}\text { Weight }(\mathrm{kg}) \text { in outpatient clinic, } \\
\text { 0-3 months }\end{array}$ & & $1.02(0.97-1.06)$ & 0.496 & -- & -- \\
\hline $\begin{array}{l}\text { Weight }(\mathrm{kg}) \text { in outpatient clinic, } \\
\text { 3-6 months }\end{array}$ & & $1.02(0.97-1.06)$ & 0.506 & -- & -- \\
\hline \multicolumn{6}{|l|}{ Performance status } \\
\hline \multicolumn{6}{|l|}{ KPS Score } \\
\hline$<50$ & $5(2-10)$ & Ref. & & Ref. & \\
\hline$\geq 50$ & $20(9-43)$ & $0.47(0.30-0.75)$ & 0.001 & $0.49(0.30-0.80)$ & 0.004 \\
\hline \multicolumn{6}{|l|}{ Prognostic Scores } \\
\hline$G P S$ & & & & & \\
\hline
\end{tabular}


Keane et al. 8

\begin{tabular}{|c|c|c|c|c|c|}
\hline \multirow[t]{2}{*}{ Variables } & \multirow{2}{*}{$\begin{array}{l}\text { Survival (weeks) } \\
\text { Median (IQR) }\end{array}$} & \multicolumn{2}{|c|}{ Univariate Cox regression } & \multicolumn{2}{|c|}{ Multivariate Cox regression } \\
\hline & & HR $(95 \%$ CI $)$ & $\bar{p}$ & HR $(95 \%$ CI $)$ & $\bar{p}$ \\
\hline$\overline{0}$ & $55(32-62)$ & Ref. & & Ref. & \\
\hline 1 & $18(7-34)$ & $2.00(0.82-4.91)$ & 0.129 & $1.91(0.77-4.75)$ & 0.162 \\
\hline 2 & $10(3-25)$ & $3.19(1.39-7.32)$ & 0.006 & $3.60(1.52-8.55)$ & 0.004 \\
\hline N/A & $12(6-30)$ & $2.26(1.01-5.08)$ & 0.049 & $2.46(1.06-5.71)$ & 0.036 \\
\hline \multicolumn{6}{|l|}{ Serum Biochemistry } \\
\hline$\overline{C R P}(\mathrm{mg} / \mathrm{L})$ & & $1.01(1.004-1.015)$ & 0.001 & $\overline{--}$ & $\overline{--}$ \\
\hline$W C C\left(\times 10^{9} / L\right)$ & & $1.06(1.01-1.10)$ & 0.011 & -- & -- \\
\hline $\operatorname{Albumin}(g / L)$ & & $0.96(0.91-1.000$ & 0.052 & -- & -- \\
\hline Haemoglobin $(\mathrm{g} / \mathrm{L})$ & & $1.01(0.99-1.03)$ & 0.322 & -- & -- \\
\hline Sodium (mmol/L) & & $0.97(0.90-1.04)$ & 0.382 & -- & -- \\
\hline \multicolumn{6}{|l|}{ Sodium (mmol/L) } \\
\hline$<135$ & $10(4-25)$ & Ref. & & -- & -- \\
\hline$\geq 135$ & $16(6-48)$ & $0.62(0.36-1.09)$ & 0.097 & -- & -- \\
\hline Potassium (mmol/L) & & $0.96(0.60-1.56)$ & 0.876 & -- & -- \\
\hline Magnesium (mmol/L) & & $1.32(0.1-15.74)$ & 0.826 & -- & -- \\
\hline Phosphate (mmol/L) & & $0.41(0.12-1.39)$ & 0.153 & -- & -- \\
\hline Adjusted calcium (mmol/L) & & $1.48(0.29-7.61)$ & 0.642 & -- & -- \\
\hline \multicolumn{6}{|l|}{ PN requirements } \\
\hline Volume (L/day) & & $1.63(1.16-2.29)$ & 0.005 & -- & -- \\
\hline \multicolumn{6}{|l|}{ Volume (L/day) } \\
\hline$<2$ & $34(5-73)$ & Ref. & & Ref. & \\
\hline$\geq 2$ & $12(6-27)$ & $1.86(1.07-3.26)$ & 0.029 & $1.66(0.93-2.98)$ & 0.087 \\
\hline Nitrogen (g/day) & & $1.04(0.96-1.12)$ & 0.386 & -- & -- \\
\hline Glucose (kcal/day) & & $1.001(1.000-1.001)$ & 0.155 & -- & -- \\
\hline Lipid (kcal/day) & & $1.00(0.99-1.001)$ & 0.664 & -- & -- \\
\hline Sodium (mmol/day) & & $1.002(0.998-1.005)$ & 0.332 & -- & -- \\
\hline Potassium (mmol/day) & & $1.009(1.001-1.016)$ & 0.025 & -- & -- \\
\hline \multicolumn{6}{|l|}{ Potassium (mmol/day) } \\
\hline$<60$ & $24(9-55)$ & Ref. & & Ref. & \\
\hline$\geq 60$ & $10(4-20)$ & $1.81(1.19-2.75)$ & 0.006 & $1.41(0.90-2.22)$ & 0.138 \\
\hline Calcium (mmol/day) & & $0.99(0.90-1.08)$ & 0.775 & -- & -- \\
\hline Magnesium (mmol/day) & & $1.03(0.99-1.07)$ & 0.103 & -- & -- \\
\hline Phosphate (mmol/day) & & $1.02(1.00-1.04)$ & 0.106 & -- & -- \\
\hline \multicolumn{6}{|l|}{ Type of PN lipid } \\
\hline Other & 12 & Ref. & & -- & -- \\
\hline SMOFLipid & 10 & $1.16(0.73-1.85)$ & 0.521 & -- & -- \\
\hline
\end{tabular}


Keane et al. 9

Figure 1. Kaplan-Meier curves for type of malignancy, HPN indication, KPS score, GPS, PN volume, and PN potassium content.

Type of Malignancy + Gynaecological $\div$ Gastrointestinal $\div$ other

HPN Indication — Bowel Obstruction — High output Ostomy — Other
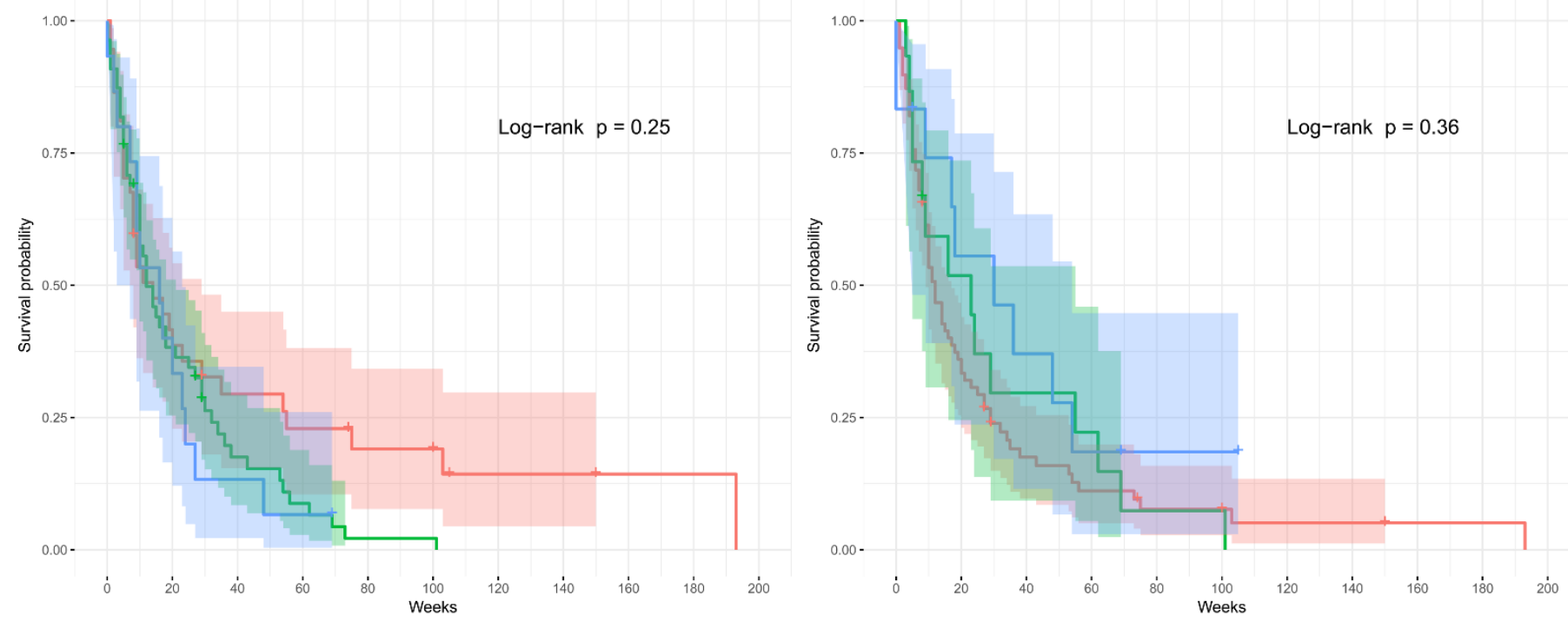

Karnofsky Performance Status + less than $50+$ equal or more than 50

Glasgow Prognostic Score $+0 \div 1+2 \div$ N/A
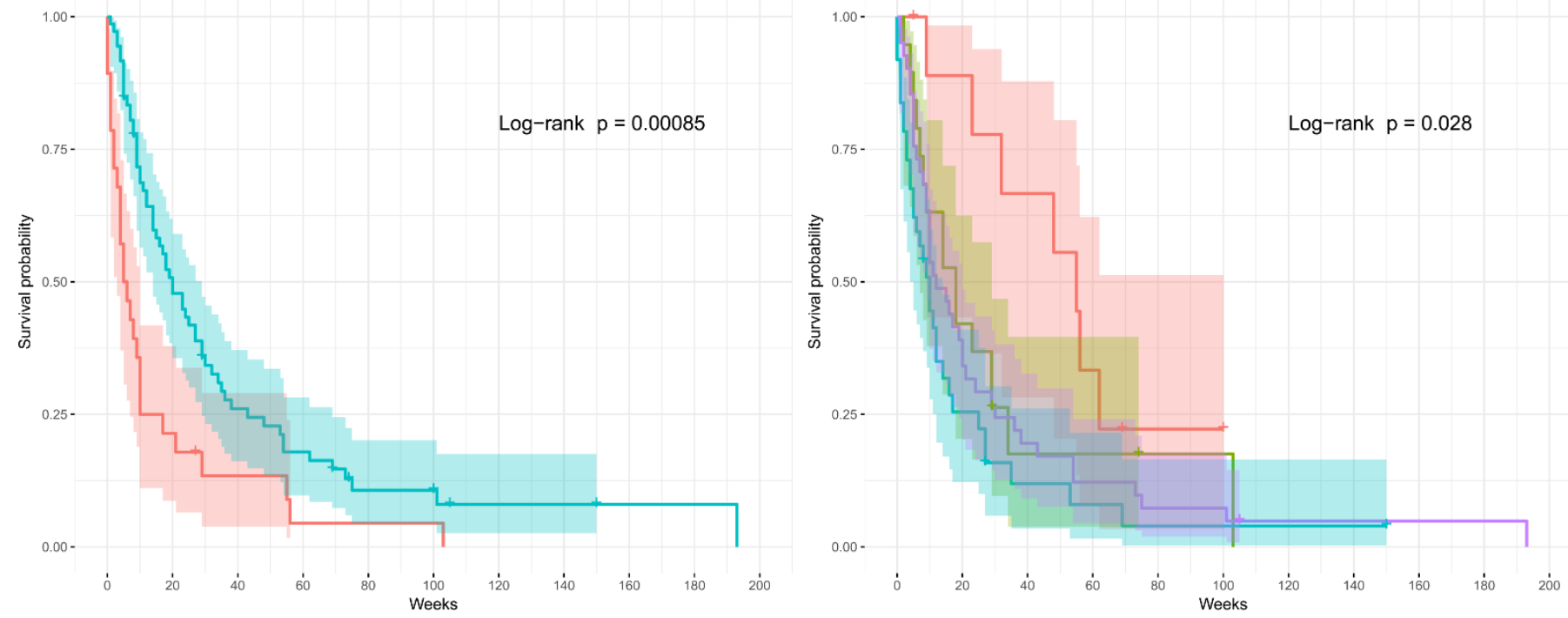

Parenteral Nutrition Volume + less than 2 Lday + equal or more than 2 L/day

Parenteral Nutrition Potassium + less than $60 \mathrm{mmol} / \mathrm{day}+$ equal or more than $60 \mathrm{mmol} / \mathrm{day}$
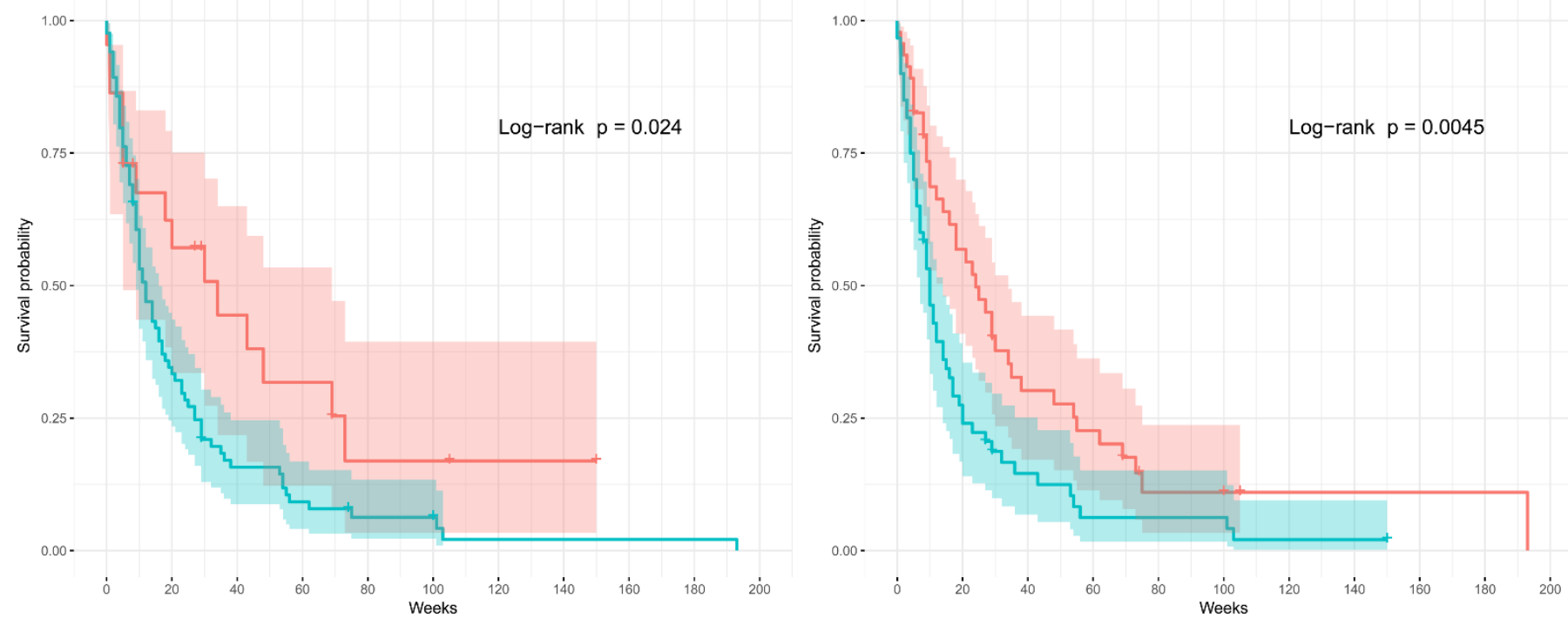
Karnofsky Performance Status and Glasgow Prognostic Score

KPS was negatively correlated with GPS, CRP and WCC, and positively correlated with albumin and weight at 0 to 3 months. GPS was strongly positively correlated with CRP and WCC, while strongly negatively correlated with albumin (Supplementary Table 1). KPS score and GPS distributions by HPN indication and type of malignancy and metastatic disease are shown in Supplementary Table 2. No significant association was noted among these variables.

\section{HPN requirements}

HPN requirements were examined according to type of malignancy, HPN indication, KPS score and GPS (Table 3). Our findings suggest a significant difference in PN volume according to HPN indication $(p=0.005)$. Patients with bowel obstruction or high output ostomy required higher volume than other indications (2328 $\mathrm{mL}, 2376 \mathrm{~mL}, 1725 \mathrm{~mL}$, respectively). There was a correlating trend between lower KPS scores with higher HPN volume requirements ( $2426 \mathrm{~mL} v s 2165 \mathrm{~mL}, p=0.066)$. Patients with bowel obstruction needed a higher content in nitrogen ( $11 \mathrm{~g}, p=0.003$ ), whilst patients with KPS score over 50 required higher PN lipid contents (607 kcal, $p=0.021)$. High output ostomies led to higher HPN sodium requirements, though this association did not reach significance ( $p=0.096$ ). PN potassium content was significantly higher in patients with bowel obstruction $(p<0.0001)$. There was a trend towards higher HPN content of calcium in patients with gynaecological malignancy or with bowel obstruction ( $p=0.072$ and $p=0.051$, respectively). 
Table 3. PN requirements according to type of malignancy, HPN indication, GPS and KPS score.

\begin{tabular}{|c|c|c|c|c|c|c|c|c|c|}
\hline & $\begin{array}{l}\text { volume } \\
\text { (mL/day) }\end{array}$ & $\begin{array}{c}\text { nitrogen } \\
\text { (g/day) }\end{array}$ & $\begin{array}{c}\text { glucose } \\
\text { (kcal/day) }\end{array}$ & $\begin{array}{c}\text { lipid } \\
\text { (kcal/day) }\end{array}$ & $\begin{array}{c}\text { sodium } \\
(\mathrm{mmol} / \mathrm{day})\end{array}$ & $\begin{array}{c}\text { potassium } \\
\text { (mmol/day) }\end{array}$ & $\begin{array}{c}\text { calcium } \\
\text { (mmol/day) }\end{array}$ & $\begin{array}{l}\text { magnesium } \\
\text { (mmol/day) }\end{array}$ & $\begin{array}{l}\text { phosphate } \\
\text { (mmol/day) }\end{array}$ \\
\hline Total & $2251 \pm 626$ & $11 \pm 3$ & $911 \pm 304$ & $573 \pm 263$ & $112 \pm 61$ & $58 \pm 26$ & $5 \pm 2$ & $10 \pm 5$ & $21 \pm 10$ \\
\hline \multicolumn{10}{|l|}{ Type of malignancy } \\
\hline Gynaecological & $2266 \pm 611$ & $11 \pm 2$ & $878 \pm 267$ & $610 \pm 267$ & $112 \pm 57$ & $56 \pm 22$ & $5 \pm 2$ & $10 \pm 6$ & $21 \pm 10$ \\
\hline Gastrointestinal & $2231 \pm 623$ & $11 \pm 3$ & $968 \pm 313$ & $576 \pm 262$ & $112 \pm 51$ & $59 \pm 25$ & $4 \pm 2$ & $10 \pm 5$ & $22 \pm 9$ \\
\hline Other & $2290 \pm 711$ & $9 \pm 3$ & $782 \pm 320$ & $477 \pm 247$ & $114 \pm 100$ & $63 \pm 40$ & $4 \pm 2$ & $10 \pm 6$ & $17 \pm 9$ \\
\hline$p$-value ${ }^{*}$ & 0.937 & 0.022 & 0.078 & 0.259 & 0.991 & 0.687 & 0.072 & 0.792 & 0.243 \\
\hline \multicolumn{10}{|l|}{ HPN indication } \\
\hline Bowel obstruction & $2328 \pm 586$ & $11 \pm 2$ & $938 \pm 255$ & $588 \pm 256$ & $113 \pm 54$ & $64 \pm 24$ & $5 \pm 2$ & $10 \pm 5$ & $21 \pm 9$ \\
\hline High output ostomy & $2376 \pm 679$ & $9 \pm 4$ & $765 \pm 434$ & $551 \pm 349$ & $136 \pm 98$ & $36 \pm 22$ & $3 \pm 2$ & $10 \pm 8$ & $16 \pm 11$ \\
\hline Other & $1725 \pm 556$ & $10 \pm 2$ & $890 \pm 380$ & $537 \pm 206$ & $85 \pm 46$ & $45 \pm 24$ & $4 \pm 2$ & $9 \pm 5$ & $21 \pm 9$ \\
\hline$p$-value ${ }^{*}$ & 0.005 & 0.003 & 0.129 & 0.764 & 0.096 & $<0.0001$ & 0.051 & 0.721 & 0.147 \\
\hline \multicolumn{10}{|l|}{ KPS Score } \\
\hline$<50$ & $2426 \pm 638$ & $11 \pm 3$ & $872 \pm 352$ & $475 \pm 304$ & $105 \pm 41$ & $63 \pm 27$ & $5 \pm 2$ & $10 \pm 5$ & $20 \pm 10$ \\
\hline$\geq 50$ & $2165 \pm 625$ & $11 \pm 2$ & $948 \pm 271$ & $607 \pm 230$ & $112 \pm 57$ & $57 \pm 26$ & $4 \pm 2$ & $10 \pm 5$ & $22 \pm 9$ \\
\hline$p$-value ${ }^{*}$ & 0.066 & 0.964 & 0.251 & 0.021 & 0.548 & 0.272 & 0.912 & 0.466 & 0.253 \\
\hline \multicolumn{10}{|l|}{ GPS } \\
\hline 0 & $1952 \pm 665$ & $9 \pm 3$ & $626 \pm 343$ & $501 \pm 325$ & $97 \pm 42$ & $44 \pm 23$ & $5 \pm 3$ & $6 \pm 5$ & $17 \pm 10$ \\
\hline 1 & $2206 \pm 597$ & $11 \pm 2$ & $964 \pm 251$ & $565 \pm 220$ & $124 \pm 64$ & $51 \pm 14$ & $5 \pm 2$ & $10 \pm 6$ & $19 \pm 9$ \\
\hline 2 & $2203 \pm 625$ & $11 \pm 3$ & $909 \pm 281$ & $552 \pm 272$ & $95 \pm 32$ & $62 \pm 28$ & $5 \pm 2$ & $9 \pm 4$ & $22 \pm 9$ \\
\hline N/A & $2392 \pm 617$ & $11 \pm 3$ & $960 \pm 307$ & $615 \pm 259$ & $126 \pm 79$ & $62 \pm 29$ & $4 \pm 2$ & $12 \pm 6$ & $23 \pm 11$ \\
\hline$p$-value ${ }^{*}$ & 0.201 & 0.058 & 0.013 & 0.570 & 0.105 & 0.122 & 0.156 & 0.020 & 0.278 \\
\hline
\end{tabular}

${ }^{*} p$-values are from ANOVA for type of malignancy, HPN indication and GPS and from $t$-test for KPS score. Values are presented as mean \pm SD. 


\section{Discussion}

We analysed the largest cohort of UK patients with advanced cancer on HPN, and examined whether survival might be predicted by demographic characteristics, medical factors, anthropometrics and nutritional status, serum blood tests, daily PN requirements, performance status and prognostic scores. Most patients required daily HPN (93.4\%). Higher daily volume and potassium requirements were associated with a worse prognosis while demographic and anthropometric characteristics did not predict survival. Interestingly, upper gastrointestinal malignancies were associated with borderline significance for higher mortality. Only CRP and WCC appeared to be significant predictors of survival whereas serum albumin and sodium showed borderline significance. These findings offer potential prognostic parameters to assist multidisciplinary teams in decision making and appropriate care planning. Predictable requirements might facilitate the use of off-the-shelf PN bags enabling faster discharge from hospital and ultimately the provision of a sustainable service despite the growing number of referrals.

Multivariate analysis identified only baseline KPS score and GPS as significant predictors of survival $(2,3$, 6, 11, 19, 34-39). In a meta-analysis, Naghibi, Smith and Elia (6) reported that patients with a higher performance status (defined as KPS greater than 50) survived longer than those with a KPS score less than 50 (median survival 183 days vs 91 days, respectively) (40). Bozzetti et al. (3, 25) described that GPS significantly correlated with three- and sixth-month survival $(p=0.001)$ and included a nomogram for predicting survival in HPN patients with advanced cancer $(25,41-44)$. Our results suggest that appropriate timely referral of advanced cancer patients for HPN should be promoted and encouraged among healthcare professionals as there seems to be a window of opportunity before a decline in performance status might nullify the beneficial effect of HPN and inappropriate therapies in vulnerable patients $(31,45)$.

The overall median length of survival in our cohort of patients receiving HPN was 14 weeks, consistent with the ESPEN guidelines of expected survival longer than 8-12 weeks, confirming appropriate indication for HPN $(1,27)$. Median duration of HPN in our study was 12.9 weeks consistent with reports from systematic reviews of 15 weeks (26). Finally, our study supports the hypothesis that a systemic inflammatory status, as assessed with higher GPS, CRP, and WCC is associated with a worse performance status (i.e. lower KPS score) and prognosis $(9,32)$.

Although cancer cachexia was prevalent in our cohort of patients, anthropometric characteristics failed to predict survival. According to the grading system by Martin et al. (10), the majority of our patients belonged to grade 4 (which predicts median survival 4.9 months). Intervention at this point is unlikely to improve cancer cachexia but rather offer the patient comfort during end of life at home rather than in hospital. This is a paramount aspect for the patients and their families and should be taken into consideration in decision making with realistic goals of the benefit of HPN in a sustainable and ethically correct process. It also has positive financial consequences in terms of healthcare system costs in palliative and end of life care. In our cohort, most patients supported with HPN, passed away in their homes or hospice (77.9\%) rather than in hospital.

This study has several limitations. Firstly, there is the possibility of collection and selection bias due to missing data. Nevertheless, this would be minimal in our study due to a sufficiently large sample size (over 100 subjects). Secondly, as a tertiary referral centre and intestinal failure unit, UCLH's practices might be significantly different compared to other hospitals without seamless access to HPN services. Finally, discharge date was used as time point for measuring variables while other research groups have used date when PN was commenced in hospital (usually 2-4 weeks earlier than discharge) (6).

In conclusion, the present study supports the use of HPN in patients with advanced cancer and offers practical tools to assist clinical teams in decision making. It shows that patients supported with HPN are likely to die at home or hospice rather than in hospital. What is apparent is the need for timely referral, since patients with 
earlier stage disease will likely have better GPS and KPS score which are associated with improved overall survival. Further education of healthcare professionals is necessary to ensure timely access to HPN for all patients with incurable cancer with an appropriate indication. Further research is needed regarding prognostic factors and quality of life in advanced cancer patients on HPN as well as the perception and experience of these patients and their families. It is imperative that HPN is used appropriately as part of an integrated sustainable palliative care plan.

\section{Acknowledgements and notes}

This study did not receive any funding. The authors would like to acknowledge the assistance of the following individuals during this research: Alastair Forbes, Shola Ajibodu, Simon O'Callaghan, Mark Samaan, Dariush Sadigh, Ivana Barnova, Sarah Obbard, Krista Murray, Lisa Smith, Michelle Babington, Emma Paulon, Klaartje (Bel) Kok. The authors also acknowledge the constructive feedback received from the reviewers which improved greatly the present paper. Financial Disclosure: The authors have no financial disclosure or conflict of interest to declare.

\section{Mailing address for reprints}

Dr Farooq Rahman

GI Services, University College London Hospitals

250 Euston Road, London NW1 2PG, United Kingdom

Tel. +44 (0) 2034567890 Ext 79311; Fax: +44 (0) 2034479217

e-mail: farooq.rahman@uclh.nhs.uk

\section{Statement}

This manuscript has not been published elsewhere and has not been submitted simultaneously for publication elsewhere. 


\section{References}

1 Arends J, Bachmann P, Baracos V, Barthelemy N, Bertz H, et al.: ESPEN guidelines on nutrition in cancer patients. Clinical Nutrition, 2016. doi: 10.1016/j.clnu.2016.07.015

2 Muscaritoli M, Molfino A, Laviano A, Rasio D, Rossi Fanelli F: Parenteral nutrition in advanced cancer patients. Crit Rev Oncol Hematol 84, 26-36, 2012. doi: 10.1016/j.critrevonc.2012.01.005

3 Bozzetti F, Santarpia L, Pironi L, Thul P, Klek S, et al.: The prognosis of incurable cachectic cancer patients on home parenteral nutrition: A multi-centre observational study with prospective follow-up of 414 patients. Annals of Oncology 25, 487-493, 2014. doi: 10.1093/annonc/mdt549

4 Bukki J, Unterpaul T, Nubling G, Jox RJ, Lorenzl S: Decision making at the end of life--cancer patients' and their caregivers' views on artificial nutrition and hydration. Support Care Cancer 22, 3287-99, 2014. doi: 10.1007/s00520-014-2337-6

5 Bozzetti F: Nutrition, hydration, and patient's preferences at the end of life. Supportive Care in Cancer 23, 1487-1488, 2015. doi: 10.1007/s00520-014-2591-7

6 Naghibi M, Smith TR, Elia M: A systematic review with meta-analysis of survival, quality of life and costeffectiveness of home parenteral nutrition in patients with inoperable malignant bowel obstruction. Clin Nutr 34, 82537, 2015. doi: 10.1016/j.clnu.2014.09.010

7 Bozzetti F, Arends J, Lundholm K, Micklewright A, Zurcher G, et al.: ESPEN Guidelines on Parenteral Nutrition: non-surgical oncology. Clin Nutr 28, 445-54, 2009. doi: 10.1016/j.clnu.2009.04.011

8 Aapro M, Arends J, Bozzetti F, Fearon K, Grunberg SM, et al.: Early recognition of malnutrition and cachexia in the cancer patient: a position paper of a European School of Oncology Task Force. Ann Oncol 25, 1492-9, 2014. doi: 10.1093/annonc/mdu085

9 Fearon KC, Voss AC, Hustead DS, Cancer Cachexia Study G: Definition of cancer cachexia: effect of weight loss, reduced food intake, and systemic inflammation on functional status and prognosis. Am J Clin Nutr 83, 1345-50, 2006

10 Martin L, Senesse P, Gioulbasanis I, Antoun S, Bozzetti F, et al.: Diagnostic criteria for the classification of cancer-associated weight loss. J Clin Oncol 33, 90-9, 2015. doi: 10.1200/JCO.2014.56.1894

11 Bozzetti F: Nutritional support of the oncology patient. Crit Rev Oncol Hematol 87, 172-200, 2013. doi: 10.1016/j.critrevonc.2013.03.006

12 Bukki J: Re: recommendations for bowel obstruction with peritoneal carcinomatosis by laval et Al. J Pain Symptom Manage 48, e5-6, 2014. doi: 10.1016/j.jpainsymman.2014.07.006

13 Soo I, Gramlich L: Use of parenteral nutrition in patients with advanced cancer. Appl Physiol Nutr Metab 33, 102-6, 2008. doi: 10.1139/H07-152

14 Mirhosseini M, Fainsinger R: Parenteral nutrition in patients with advanced cancer \#190. J Palliat Med 12, 2601, 2009. doi: 10.1089/jpm.2009.9653

15 Laval G, Marcelin-Benazech B, Guirimand F, Chauvenet L, Copel L, et al.: Recommendations for bowel obstruction with peritoneal carcinomatosis. J Pain Symptom Manage 48, 75-91, 2014 . doi: 10.1016/j.jpainsymman.2013.08.022

16 Baxter JP, McKee RF: The Scottish home parenteral nutrition managed clinical network: one year on. Clin Nutr 22, 501-4, 2003

17 King LA, Carson LF, Konstantinides N, House MS, Adcock LL, et al.: Outcome assessment of home parenteral nutrition in patients with gynecologic malignancies: what have we learned in a decade of experience? Gynecol Oncol 51, 377-82, 1993. doi: 10.1006/gyno.1993.1307

18 Aria Guerra E, Cortes-Salgado A, Mateo-Lobo R, Nattero L, Riveiro J, et al.: Role of Parenteral Nutrition in Oncologic Patients with Intestinal Occlusion and Peritoneal Carcinomatosis. Nutr Hosp 32, 1222-7, 2015. doi: 10.3305/nh.2015.32.3.9184 
Scolapio JS, Fleming CR, Kelly DG, Wick DM, Zinsmeister AR: Survival of Home Parenteral NutritionTreated Patients: 20 Years of Experience at the Mayo Clinic. Mayo Clinic Proceedings 74, 217-222, 1999. doi: 10.4065/74.3.217

20 Bozzetti F, Cozzaglio L, Biganzoli E, Chiavenna G, De Cicco M, et al.: Quality of life and length of survival in advanced cancer patients on home parenteral nutrition. Clin Nutr 21, 281-8, 2002

21 Duerksen DR, Ting E, Thomson P, McCurdy K, Linscer J, et al.: Is there a role for TPN in terminally ill patients with bowel obstruction? Nutrition 20, 760-763, 2004. doi: 10.1016/j.nut.2004.05.010

22 Hoda D, Jatoi A, Burnes J, Loprinzi C, Kelly D: Should patients with advanced, incurable cancers ever be sent home with total parenteral nutrition? Cancer 103, 863-868, 2005. doi: 10.1002/cncr.20824

23 Brard L, Weitzen S, Strubel-Lagan SL, Swamy N, Gordinier ME, et al.: The effect of total parenteral nutrition on the survival of terminally ill ovarian cancer patients. Gynecologic Oncology 103, 176-180, 2006. doi: 10.1016/j.ygyno.2006.02.013

24 Chermesh I, Mashiach T, Amit A, Haim N, Papier I, et al.: Home parenteral nutrition (HTPN) for incurable patients with cancer with gastrointestinal obstruction: Do the benefits outweigh the risks? Medical Oncology 28, 83-88, 2011. doi: 10.1007/s12032-010-9426-2

25 Bozzetti F, Cotogni P, Lo Vullo S, Pironi L, Giardiello D, et al.: Development and validation of a nomogram to predict survival in incurable cachectic cancer patients on home parenteral nutrition. Annals of Oncology 26, 2335-2340, 2015. doi: $10.1093 /$ annonc/mdv365

26 Fragkos K, Fini L, Keane N, Kwok H, Paulon E, et al.: PTU-109 Home parenteral nutrition in patients with advanced cancer: a systematic review. Gut 66, A104-A105, 2017. doi: 10.1136/gutjnl-2017-314472.204

27 Staun M, Pironi L, Bozzetti F, Baxter J, Forbes A, et al.: ESPEN Guidelines on Parenteral Nutrition: home parenteral nutrition (HPN) in adult patients. Clin Nutr 28, 467-79, 2009. doi: 10.1016/j.clnu.2009.04.001

28 Pironi L, Ruggeri E, Tanneberger S, Giordani S, Pannuti F, et al.: Home artificial nutrition in advanced cancer. Journal of the Royal Society of Medicine 90, 597-603, 1997

29 Karnofsky DA, Abelmann WH, Craver LF, Burchenal JH: The use of the nitrogen mustards in the palliative treatment of carcinoma.With particular reference to bronchogenic carcinoma. Cancer 1, 634-656, 1948. doi: 10.1002/1097-0142(194811)1:4<634::aid-cncr2820010410>3.0.co;2-1

30 Schag CC, Heinrich RL, Ganz PA: Karnofsky performance status revisited: reliability, validity, and guidelines. J Clin Oncol 2, 187-93, 1984

31 Hui D: Prognostication of Survival in Patients With Advanced Cancer: Predicting the Unpredictable? Cancer Control 22, 489-97, 2015

32 Fearon K, Strasser F, Anker SD, Bosaeus I, Bruera E, et al.: Definition and classification of cancer cachexia: an international consensus. The Lancet Oncology 12, 489-495, 2011. doi: 10.1016/s1470-2045(10)70218-7

33 Tian H, Yao X, Zeng R, Sun R, Tian H, et al.: Safety and efficacy of a new parenteral lipid emulsion (SMOF) for surgical patients: a systematic review and meta-analysis of randomized controlled trials. Nutr Rev 71, 815-21, 2013. doi: 10.1111/nure.12053

34 Evans C, McCarthy M: Prognostic uncertainty in terminal care: can the Karnofsky index help? Lancet 1, 12046,1985

35 Matsunuma R, Tanbo Y, Asai N, Ohkuni Y, Watanabe S, et al.: Prognostic factors in patients with terminal stage lung cancer. J Palliat Med 17, 189-94, 2014. doi: 10.1089/jpm.2013.0448

36 Schonwetter RS, Robinson BE, Ramirez G: Prognostic factors for survival in terminal lung cancer patients. $J$ Gen Intern Med 9, 366-71, 1994

37 Vigano A, Dorgan M, Buckingham J, Bruera E, Suarez-Almazor ME: Survival prediction in terminal cancer patients: a systematic review of the medical literature. Palliat Med 14, 363-74, 2000

38 Keane N, Fragkos KC, Patel PS, Obbard S, Murray K, et al.: MON-P068: An Updated Audit of Home Parenteral Nutrition (HPN) in Palliative Oncology Patients - A UK Single Centre Case Series with Survival Analysis. Clinical Nutrition 35, S178, 2016. doi: 10.1016/s0261-5614(16)30702-6

39 Glare P, Sinclair C, Downing M, Stone P, Maltoni M, et al.: Predicting survival in patients with advanced disease. Eur J Cancer 44, 1146-56, 2008. doi: 10.1016/j.ejca.2008.02.030 
40 McMillan DC: The systemic inflammation-based Glasgow Prognostic Score: a decade of experience in patients with cancer. Cancer Treat Rev 39, 534-40, 2013. doi: 10.1016/j.ctrv.2012.08.003

41 Brown DJ, Milroy R, Preston T, McMillan DC: The relationship between an inflammation-based prognostic score (Glasgow Prognostic Score) and changes in serum biochemical variables in patients with advanced lung and gastrointestinal cancer. J Clin Pathol 60, 705-8, 2007. doi: 10.1136/jcp.2005.033217

42 Proctor MJ, Talwar D, Balmar SM, O'Reilly DS, Foulis AK, et al.: The relationship between the presence and site of cancer, an inflammation-based prognostic score and biochemical parameters. Initial results of the Glasgow Inflammation Outcome Study. Br J Cancer 103, 870-6, 2010. doi: 10.1038/sj.bjc.6605855

43 Pantano Nde P, Paiva BS, Hui D, Paiva CE: Validation of the Modified Glasgow Prognostic Score in Advanced Cancer Patients Receiving Palliative Care. J Pain Symptom Manage 51, 270-7, 2016. doi: 10.1016/j.jpainsymman.2015.09.010

44 Douglas E, McMillan DC: Towards a simple objective framework for the investigation and treatment of cancer cachexia: the Glasgow Prognostic Score. Cancer Treat Rev 40, 685-91, 2014. doi: S0305-7372(13)00260-0 [pii]

10.1016/j.ctrv.2013.11.007

45 Maltoni M, Caraceni A, Brunelli C, Broeckaert B, Christakis N, et al.: Prognostic factors in advanced cancer patients: evidence-based clinical recommendations--a study by the Steering Committee of the European Association for Palliative Care. J Clin Oncol 23, 6240-8, 2005. doi: 10.1200/JCO.2005.06.866 


\section{Supplementary Materials}

\section{Supplementary Tables}

Supplementary Table 1. Spearman correlations between variables. Only correlations with $p<0.05$ are shown. Censored cases were not included in this analysis.

\begin{tabular}{llccc}
\hline Variable 1 & Variable 2 & Spearman's rho & $\boldsymbol{p}$ & $\mathbf{N}$ \\
\hline Survival & CRP $(\mathbf{m g} / \mathbf{L})$ & -0.484 & $<0.001$ & 55 \\
Survival & WCC $(\mathbf{x ~ 1 0 9 / L )}$ & -0.300 & 0.027 & 54 \\
Survival & Albumin (g/L) & 0.338 & 0.012 & 55 \\
Survival & PN Potassium (mmol/day) & -0.247 & 0.016 & 94 \\
Survival & KPS Score & 0.374 & $<0.001$ & 88 \\
Survival & GPS & -0.444 & 0.001 & 55 \\
\hline KPS Score & Age & -0.276 & 0.010 & 87 \\
KPS Score & Weight in clinic, months 0-3 & 0.401 & 0.031 & 29 \\
KPS Score & CRP (mg/L) & -0.329 & 0.017 & 52 \\
KPS Score & WCC (x 109/L) & -0.523 & $<0.001$ & 51 \\
KPS Score & Albumin (g/L) & 0.288 & 0.038 & 52 \\
KPS Score & Haemoglobin (g/L) & -0.294 & 0.038 & 50 \\
KPS Score & PN Phosphate (mmol/day) & 0.230 & 0.032 & 87 \\
KPS Score & GPS & -0.281 & 0.043 & 52 \\
\hline GPS & CRP (mg/L) & 0.703 & $<0.001$ & 55 \\
GPS & WCC (x 109/L) & 0.529 & $<0.001$ & 54 \\
GPS & Albumin (g/L) & -0.683 & $<0.001$ & 55 \\
\hline
\end{tabular}

Supplementary Table 2. KPS score and GPS distribution according to type of malignancy, metastasis, and HPN indication.

\begin{tabular}{lccc|ccccc}
\hline & \multicolumn{3}{c|}{ KPS Score } & \multicolumn{5}{c}{ GPS } \\
\cline { 2 - 8 } & $<\mathbf{5 0}$ & $\mathbf{2 5 0}$ & $\boldsymbol{p}^{*}$ & $\mathbf{0}$ & $\mathbf{1}$ & $\mathbf{2}$ & $\mathbf{N} / \mathbf{A}$ & $\boldsymbol{p}^{*}$ \\
\hline Type of & & & & & & & \\
malignancy & & & & & & & \\
\hline Gynaecological & $9(25.7 \%)$ & $26(74.3 \%)$ & 0.931 & $4(10.8 \%)$ & $7(18.9 \%)$ & $11(29.7 \%)$ & $15(40.5 \%)$ & 0.968 \\
Gastrointestinal & $15(29.4 \%)$ & $36(70.6 \%)$ & & $4(7.3 \%)$ & $10(18.2 \%)$ & $20(36.4 \%)$ & $21(38.2 \%)$ \\
Other & $4(28.6 \%)$ & $10(71.4 \%)$ & & $2(13.3 \%)$ & $2(13.3 \%)$ & $6(40.0 \%)$ & $5(33.3 \%)$ \\
\hline Metastasis & & & & & & & & \\
\hline No & $4(21.1 \%)$ & $15(78.9 \%)$ & 0.454 & $3(15.0 \%)$ & $2(10.0 \%)$ & $8(40.0 \%)$ & $7(35.0 \%)$ & 0.582 \\
Yes & $24(21.1 \%)$ & $57(70.4 \%)$ & & $7(8.0 \%)$ & $17(19.5 \%)$ & $29(33.3 \%)$ & $34(39.1 \%)$ \\
\hline Indication for HPN & & & & & & & & \\
\hline Bowel Obstruction & $21(29.2 \%)$ & $51(70.8 \%)$ & 0.820 & $5(6.4 \%)$ & $13(16.7 \%)$ & $27(34.6 \%)$ & $33(42.3 \%)$ & 0.430 \\
High output & $3(21.4 \%)$ & $11(78.6 \%)$ & & $2(13.3 \%)$ & $4(26.7 \%)$ & $5(33.3 \%)$ & $4(26.7 \%)$ \\
ostomy & & & & & & & \\
Other & $3(25.0 \%)$ & $9(75.0 \%)$ & & $3(25.0 \%)$ & $2(16.7 \%)$ & $3(25.0 \%)$ & $4(33.3 \%)$ \\
\hline
\end{tabular}




\section{Supplementary Figures}

Supplementary Figure 1. Kaplan-Meier Curve for age, gender, type of malignancy, metastasis, surgery, and chemotherapy.

Age (years) - under $50-50-64-$ over 65
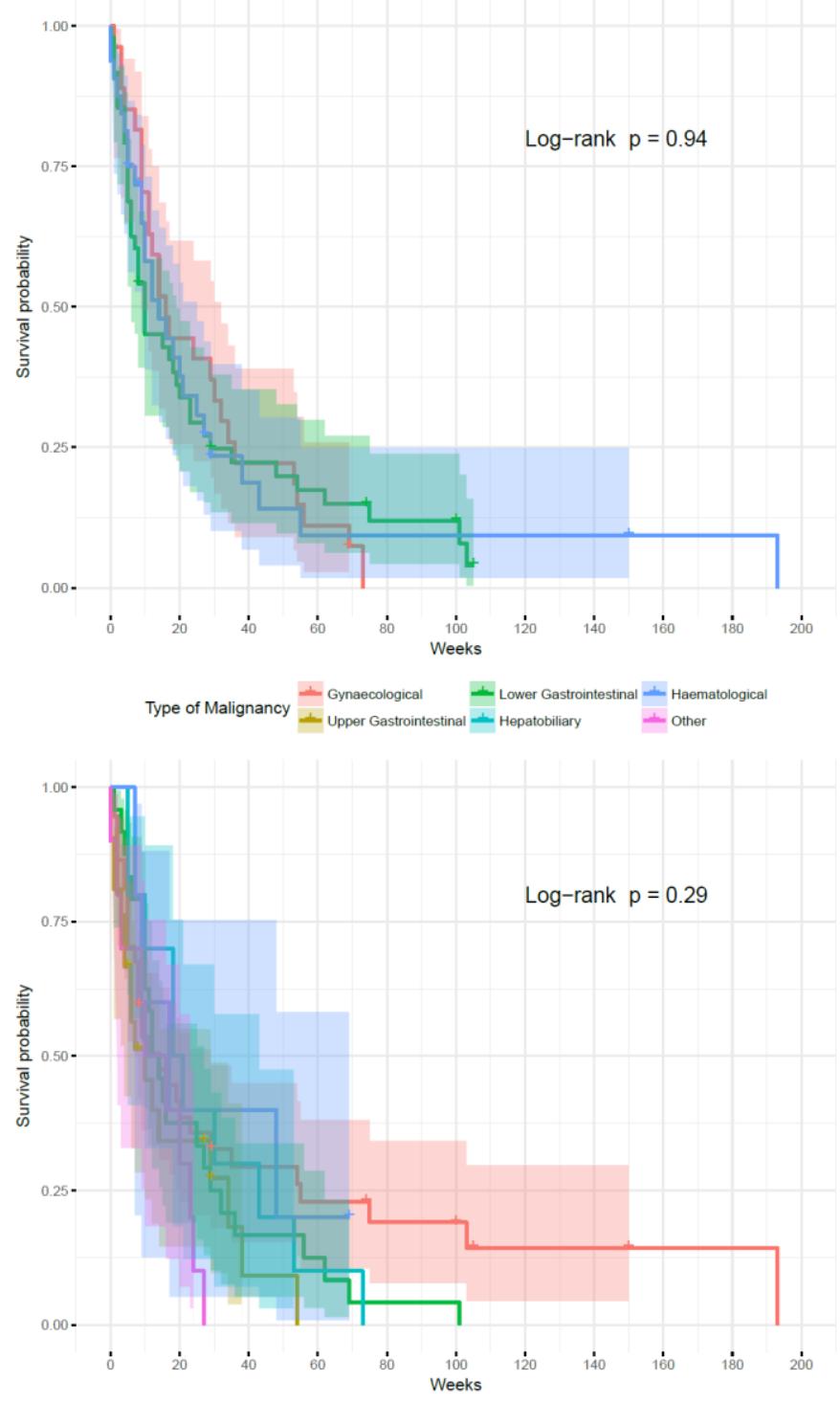

Surgery $\perp$ No - Yes

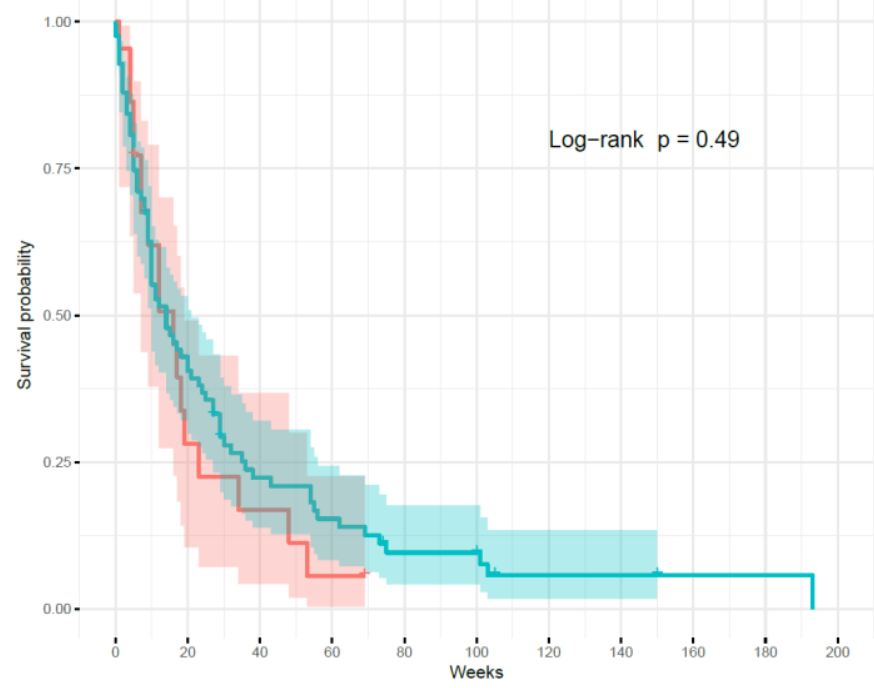

Gender + Female + Male

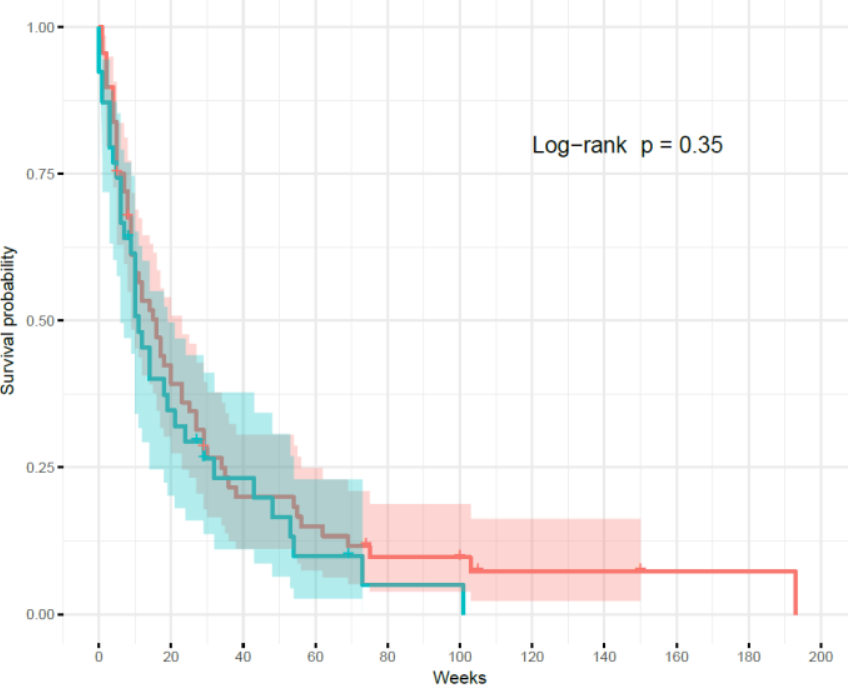

Metastasis - No - Yes

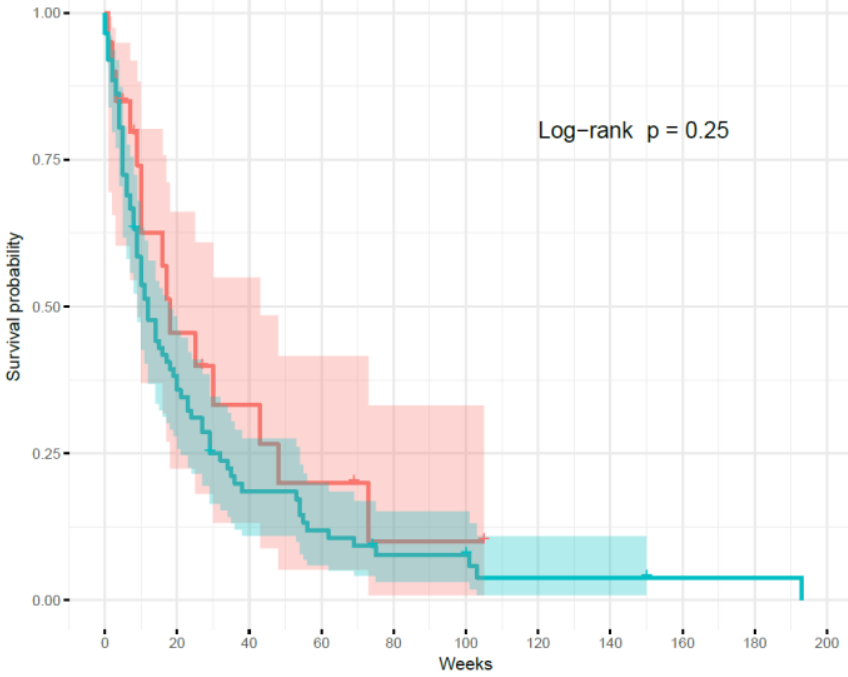

Chemotherapy \pm No $\perp$ Yes

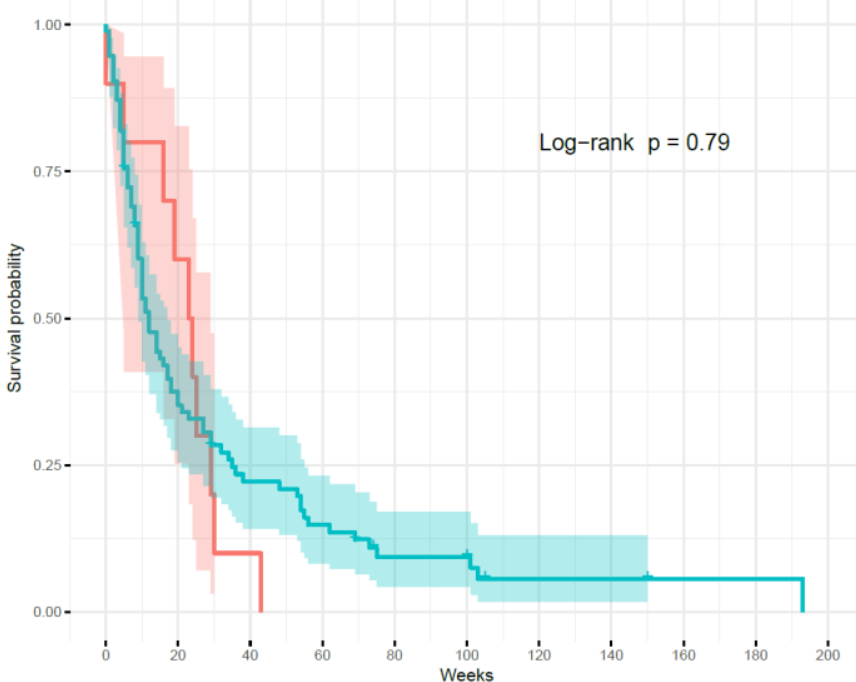


Keane et al. 19

Supplementary Figure 2. Kaplan-Meier Curve for radiotherapy, weight loss, BMI, cancer cachexia, parenteral nutrition lipid, and serum sodium.
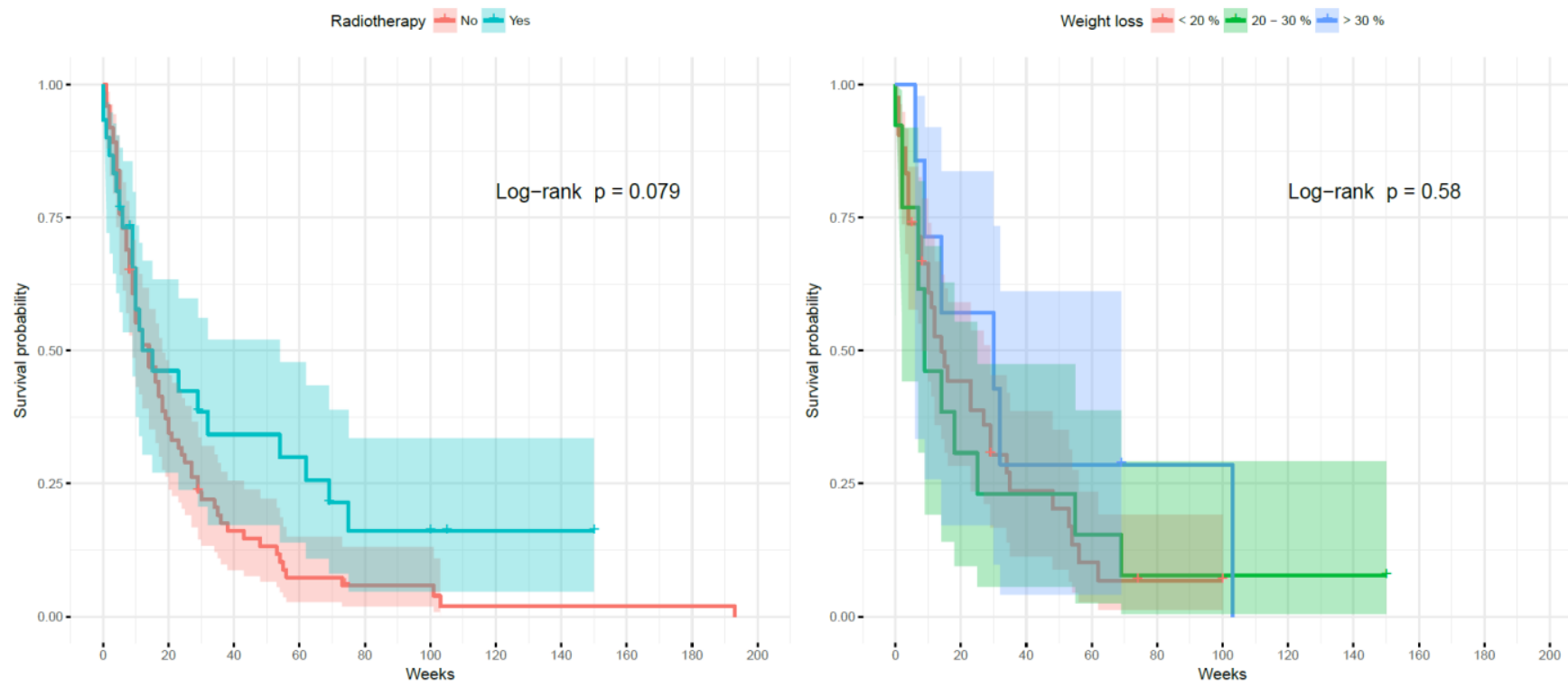

$\mathrm{BMI}=$ equal or less than $18.5 \mathrm{~kg} / \mathrm{m} 2$ - more than $18.5 \mathrm{~kg} / \mathrm{m} 2$

Cancer Cachexia - No $^{-}$Yes
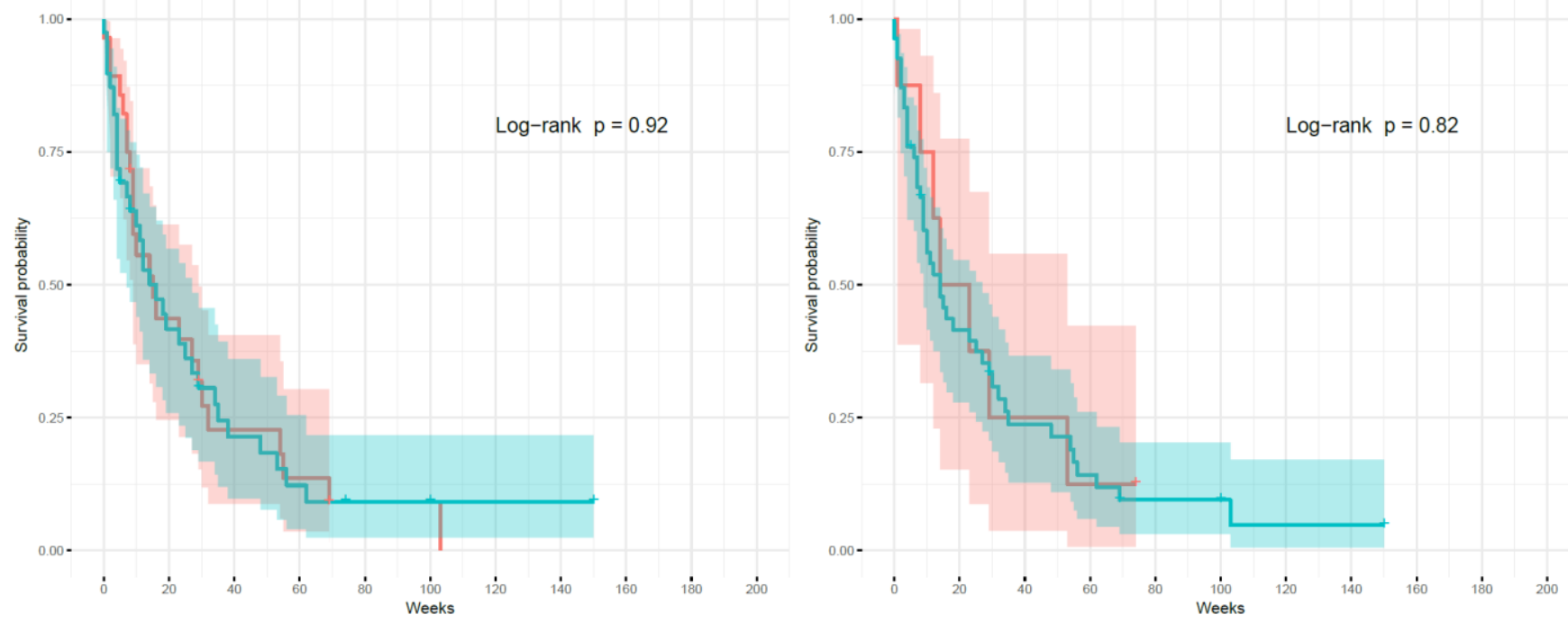

Parenteral Nutrition Lipid - other $\perp$ SMOF

Serum Sodium $\perp$ less than 135 mmoll $\perp$ equal or more than 135 mmoll
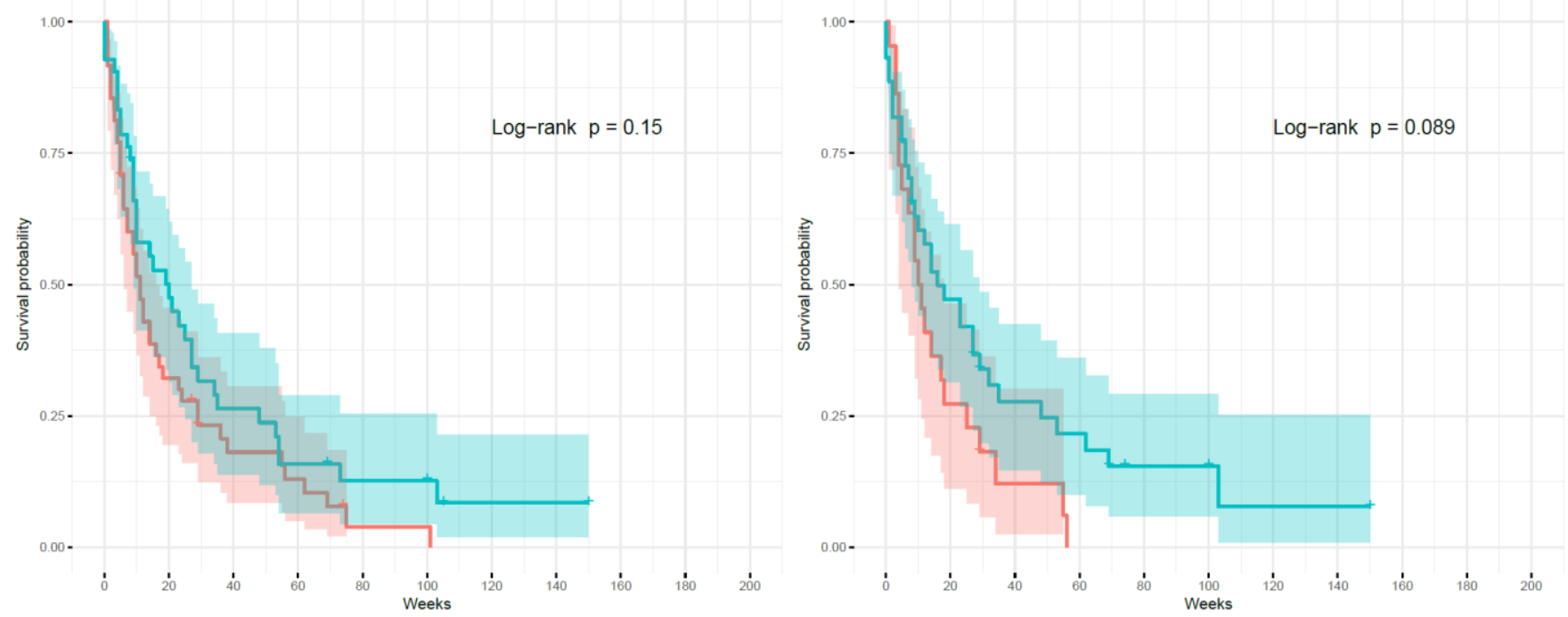